\title{
Nivel de actividad física medida a través del cuestionario internacional de actividad física en población chilena
}

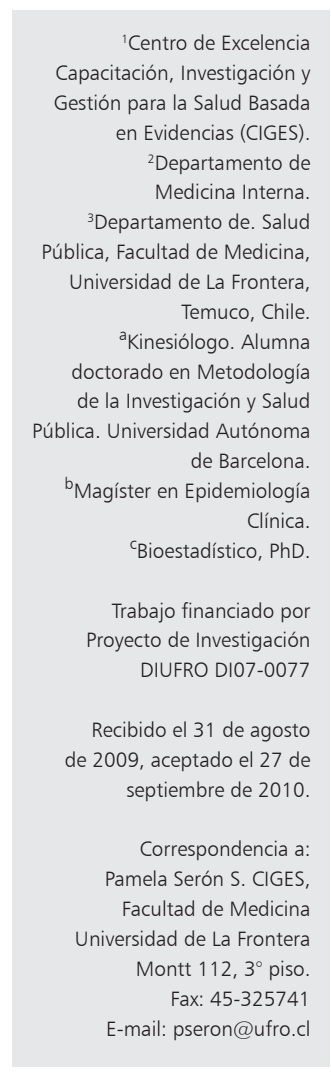

\author{
PAMELA SERÓN ${ }^{1,2, a,}$, SERGIO MUÑOZ ${ }^{2,3, c,}$ \\ FERNANDO LANAS ${ }^{1,2, b}$
}

Background: Physical activity plays a crucial role in the protection against cardiovascular diseases. Aim: To assess the level of physical activity in a group of subjects living in urban Temuco. Material and Methods: Cross sectional study in a random sample of 1091 women aged $52 \pm 10$ years and 444 men aged $54 \pm 10$ years, living in Temuco, Chile. The level of physical activity was measured using the long form of the International Physical Activity Questionnaire (IPAQ). Age, gender, educational and socioeconomic level were also determined in study subjects. Results: Median energy expenditure was 2150 and 1600 MET-minutel week in men and women, respectively $(p=0,001)$. It decreased with age from a median of 1965 MET-minute/ week in those younger than 50 years old to 1647 MET-minute/ week among subjects aged between 51 and 60 years old and to 1485 MET-minute/ week among those older than 60 years. $(p=0,001)$. The frequency of high, moderate and low physical activity levels were 15.6, 66 and $18.4 \%$ respectively. These levels were associated with gender, age, educational and socioeconomic level. Conclusions: There was a high frequency of low and moderate levels of physical activity in the urban population of Temuco, associated with female gender, advanced age and middle socioeconomic level.

(Rev Med Chile 2010; 138: 1232-1239).

Key words: Cardiovascular diseases; Motor activity; Social class.

L a actividad física es definida como cualquier movimiento corporal producido por los músculos esqueléticos y que resultan en gasto energético ${ }^{1}$. En el mundo, el problema de la inactividad física o sedentarismo tiene una alta prevalencia; en un estudio de casos y controles, que evaluó los factores de riesgo en 52 países, se comunicó $85,73 \%$ de inactividad física entre los que tuvieron un evento coronario y $80,72 \%$ entre los que no lo tuvieron ${ }^{2}$; este mismo estudio reportó $78 \%$ de inactividad física en Latinoamérica $^{3}$. En Chile, el sedentarismo es prácticamente la regla en la población, siendo bajo el porcentaje de aquellos que realizan vida física activa. La Encuesta Nacional de Salud reporta una prevalencia de sedentarismo total de $89,4 \%$, siendo de $90,8 \%$ para mujeres y de $87,9 \%$ para hombres ${ }^{4}$.

Si bien es cierto que la mayoría de los estudios hablan de prevalencias por sobre $70 \%$ de inactividad física, existe amplia variación de resultados lo que puede deberse a la diversidad de instrumentos utilizados $^{5}$ y puntos de corte inconsistentes ${ }^{6}$. Dependiendo del instrumento de evaluación puede clasificarse los niveles de actividad física en escalas dicotómicas (físicamente inactivo o sedentario/ físicamente activo), ordinales (físicamente inactivo o sedentario/moderadamente activo/físicamente activo) o continuas (kilocalorías, METS). La mayoría de los estudios nacionales ha calificado de sedentarios a quienes no practican actividad física 
al menos 30 minutos seguidos por tres veces a la semana y fuera del trabajo, es decir dicotomizando un fenómeno que es esencialmente continuo ${ }^{7-10}$. La Organización Mundial de la Salud (OMS) establece que las personas tienen la oportunidad de mantenerse físicamente activas en cuatro sectores principales de la vida diaria: el trabajo, el transporte, las tareas domésticas y el tiempo libre o de ocio ${ }^{11}$; recomendando utilizar instrumentos de medición capaces de recoger información en todas estas dimensiones.

Entre los instrumentos existentes para medir la actividad física se puede mencionar el Cuestionario Internacional de Actividad Física (IPAQ), el cual ha sido utilizado en diversos estudios internacionales y se ha evaluado su validez y confiabilidad sugiriéndose su uso en diferentes países e idiomas ${ }^{12,13}$, además de ser propuesto por la OMS como un instrumento a utilizarse para vigilancia epidemiológica a nivel poblacional, dado que se ha puesto a prueba en 24 países y actualmente se emplea en varias redes regionales ${ }^{14}$. Este instrumento aporta información sobre gasto energético estimado en 24 horas, en las distintas áreas de la vida diaria; tiene la ventaja de ser aplicable a grandes muestras de distintos niveles socioeconómicos dada su simplicidad tanto en la administración como en la obtención de los puntajes.

En este contexto, el presente estudio tiene como objetivo determinar el nivel de gasto energético en distintas actividades de la vida diaria y la frecuencia de distintos niveles de actividad física en una población chilena, a través del IPAQ, además de explorar la asociación con variables sociodemográficas.

\section{Material y Método}

En un estudio de corte transversal se seleccionaron, a través de un muestreo aleatorio por conglomerado, sujetos de ambos sexos entre 35 y 70 años, provenientes de la población urbana de Temuco en la IX región de Chile. Se determinó Nivel de Actividad Física a través del IPAQ (versión en español de 2002) en su versión larga dado que es el estándar a partir del cuál se ha elaborado la versión corta, además del sexo, edad, nivel socioeconómico (NSE) y educacional.

El IPAQ mide el nivel de actividad física a través de preguntas en cuatro dominios: laboral, domés- tico, de transporte y del tiempo libre ${ }^{15}$. El indicador de actividad física se expresa tanto de manera continua, en MET-minutos/semana, como de manera categórica, clasificando el nivel de actividad física en bajo, moderado o alto. Los METs son una forma de calcular los requerimientos energéticos, son múltiplos de la tasa metabólica basal y la unidad utilizada, MET-minuto, se calcula multiplicando el MET correspondiente al tipo de actividad por los minutos de ejecución de la misma en un día o en una semana, es así como en el presente trabajo se expresa en MET-minuto/semana ${ }^{16}$. El nivel educacional formal incluyó las alternativas: ninguno, básico, escuela técnica o universidad cuando los ciclos hayas sido completos. Para determinar el NSE se utilizó el método ESOMAR el cual se basa en el nivel educacional y la categoría ocupacional del principal sostenedor del hogar ${ }^{4,17}$ clasificando el NSE por letras en A, B, CA, CB, D y E, las que fueron agrupadas en 3 categorías: NSE alto (A y $\mathrm{B})$, medio (CA y CB) y bajo (D y E).

Para el cálculo del tamaño de muestra se consideró como antecedente la prevalencia de sedentarismo de 38,0\% en el estudio de Bustos et $\mathrm{al}^{18}$, un nivel de confianza de $95 \%$ y un error de muestreo absoluto de 3. El tamaño de muestra resulta en 1.005 sujetos; como se trata de una muestra por conglomerado, se estimó un efecto de diseño de 1,2 , lo cual da como resultado final una estimación del tamaño de muestra mínimo de 1.206 sujetos.

Se calculó la frecuencia en porcentajes de los niveles de actividad física alto, moderado y bajo (Tabla 1). Se realizó, también, un análisis comparativo de los niveles de actividad física según diversos subgrupos de la muestra estudiada, como sexo, edad y niveles educacional y socioeconómico, aplicando el método de ajuste de comparaciones múltiples de Bonferroni. Para el análisis del gasto energético y dado que la distribución de los datos no es simétrica, se utilizan las medianas y el recorrido intercuartílico. El protocolo fue aprobado por el Comité de Ética Científico del Servicio de Salud Araucanía Sur, los participantes firmaron un consentimiento informado.

\section{Resultados}

Se reclutaron 1.535 sujetos, de los cuales $71,1 \%$ eran mujeres, el promedio de edad en las mujeres fue de 52,3 \pm 9,8 años y en los hombres de 
Tabla 1. Clasificación de los niveles de actividad física según los criterios establecidos por el IPAQ

\begin{tabular}{|c|c|}
\hline Nivel de actividad física alto & $\begin{array}{l}\rightarrow \text { Reporte de } 7 \text { días en la semana de cualquier combinación de caminata, o activi- } \\
\text { dades de moderada o alta intensidad logrando un mínimo de } 3.000 \text { MET-min/ } \\
\text { semana; } \\
\rightarrow \text { o cuando se reporta actividad vigorosa al menos } 3 \text { días a la semana alcanzando } \\
\quad \text { al menos } 1.500 \mathrm{MET}-\mathrm{min} / \mathrm{semana}\end{array}$ \\
\hline Nivel de actividad física moderado & $\begin{array}{l}\rightarrow \text { Reporte de } 3 \text { o más días de actividad vigorosa por al menos } 20 \text { minutos diarios; } \\
\rightarrow \text { o cuando se reporta } 5 \text { o más días de actividad moderada y/o caminata al menos } \\
\quad 30 \text { minutos diarios; } \\
\rightarrow \text { o cuando se describe } 5 \text { o más días de cualquier combinación de caminata y activi- } \\
\text { dades moderadas o vigorosas logrando al menos } 600 \mathrm{MET} \text {-min/semana }\end{array}$ \\
\hline Nivel de actividad física bajo & $\begin{array}{l}\rightarrow \text { Se define cuando el nivel de actividad física del sujeto no esté incluido en las } \\
\text { categorías alta o moderada }\end{array}$ \\
\hline
\end{tabular}

Tabla 2. Distribución del gasto energético (en MET-minutos/semana) por actividad, según sexo

\begin{tabular}{|lcccccc|}
\hline & \multicolumn{2}{c}{ MET-minutos/semana Femenino } & \multicolumn{2}{c|}{ MET-minutos/semana Masculino } \\
Actividad & $\begin{array}{c}\text { Percentil } \\
\mathbf{2 5 \%}\end{array}$ & Mediana & $\begin{array}{c}\text { Percentil } \\
\mathbf{7 5 \%}\end{array}$ & $\begin{array}{c}\text { Percentil } \\
\mathbf{2 5 \%}\end{array}$ & $\begin{array}{c}\text { Mediana } \\
\text { Percentil } \\
\mathbf{7 5 \%}\end{array}$ \\
Trabajo & 0 & 353 & 2.280 & 0 & 597 & 4.800 \\
Transporte & 0 & 198 & 495 & 0 & 231 & 231 \\
Hogar & 0 & 0 & 735 & 0 & 41 & 855 \\
Recreación & 0 & 0 & 396 & 0 & 0 & 396 \\
Total & 480 & 1.600 & 4.284 & 492 & 2.150 & 7.730 \\
\hline
\end{tabular}

$53,5 \pm 9,8 ; 37,3 \%$ de la muestra estaban laboralmente activos.

El gasto energético expresado en MET-minuto/ semana fue calculado para cada tipo de actividad y para la suma de todas, siendo las medianas de gasto energético de $1.600 \mathrm{MET}$-minuto/semana para las mujeres y de 2.150 para los hombres. Los rangos intercuartílicos se presentan en la Tabla 2 representándose gráficamente la tendencia por tipo de actividad de acuerdo a sexo en la Figura 1, destacándose un gasto energético significativamente mayor en hombres que en mujeres $(\mathrm{p}<0,001)$, exceptuando las actividades del tiempo libre y las realizadas como tareas del hogar en que en ambos sexos tienen bajos niveles de actividad.

Considerando los grupos de edad: menores de 50 años, sujetos entre 51 y 60 años y mayores de 60 años, las medianas fueron de 1.965, $1.647 \mathrm{y}$ 1.485 MET-minuto/semana respectivamente ( $\mathrm{p}$ $<0,001)$. La tendencia por tipo de actividad de acuerdo a grupo de edad se presenta en la Figura 2 y los rangos intercuartílicos en la Tabla 3. El grupo de menos de 50 años mostró un nivel de actividad significativamente mayor en lo correspondiente a actividades del trabajo y transporte, al contrario de lo que sucede en las actividades relacionadas a tareas del hogar y del tiempo libre donde el gasto energético es menor que los otros grupos de edad.

La frecuencia de los distintos niveles de actividad física fue significativamente distinta entre hombres y mujeres, observándose en las mujeres una mayor frecuencia del nivel de actividad física moderado con respecto de los hombres y una menor frecuencia del nivel de actividad física bajo. Con respecto a la distribución por grupo de edad, es destacable cómo disminuye la frecuencia del nivel alto de actividad física a medida que aumenta la edad a la vez que el nivel moderado de actividad aumenta. Los datos se presentan en la Tabla 4.

$\mathrm{Al}$ analizar los niveles de actividad física considerando el nivel educacional se observan frecuen- 
Actividad física en población urbana - P. Serón et al

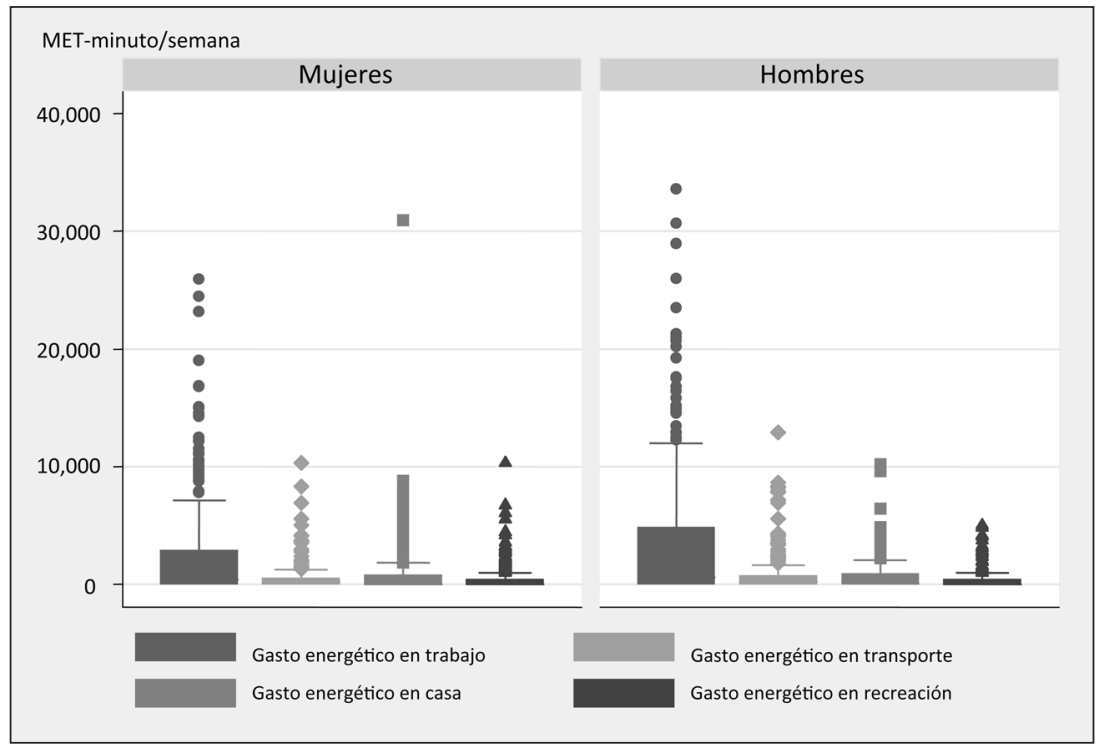

Figura 1. Distribución del gasto energético (en MET-minuto/ semana) por tipo de actividad y según sexo.

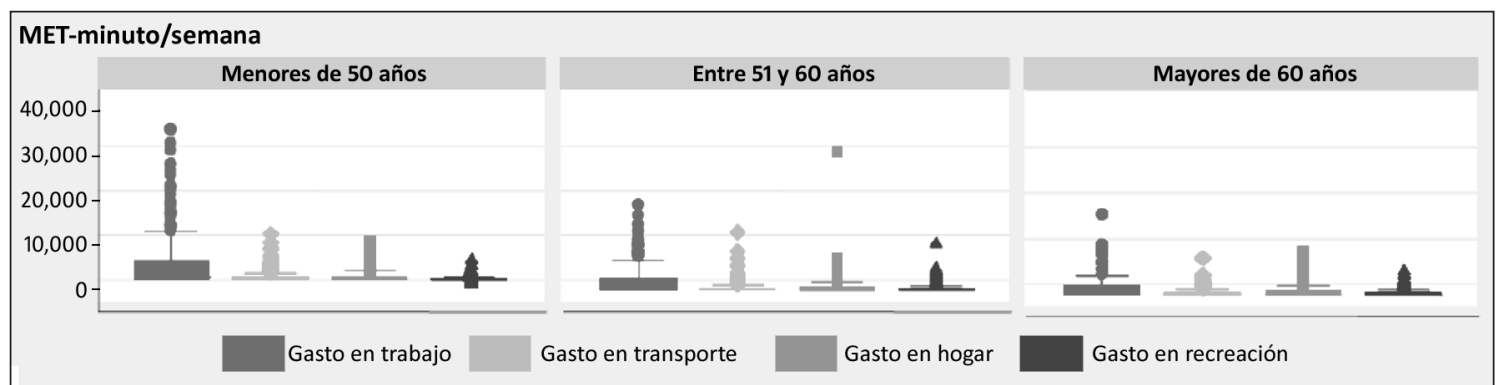

Figura 2. Distribución del gasto energético (en MET-minuto/semana) por tipo de actividad y según grupos de edad.

Tabla 3. Distribución del gasto energético (en MET-minutos/semana) por actividad, según grupos de edad

\begin{tabular}{|c|c|c|c|c|c|c|c|c|c|}
\hline \multirow[b]{2}{*}{ Actividad } & \multicolumn{3}{|c|}{$\begin{array}{l}\text { MET-minutos/semana } \\
<=50 \text { años }\end{array}$} & \multicolumn{3}{|c|}{$\begin{array}{l}\text { MET-minutos/semana } \\
\text { 51-60 años }\end{array}$} & \multicolumn{3}{|c|}{$\begin{array}{l}\text { MET-minutos/semana } \\
\text { > } 60 \text { años }\end{array}$} \\
\hline & $\begin{array}{l}\text { Percentil } \\
25 \%\end{array}$ & Mediana & $\begin{array}{l}\text { Percentil } \\
\mathbf{7 5} \%\end{array}$ & $\begin{array}{l}\text { Percentil } \\
\mathbf{2 5 \%}\end{array}$ & Mediana & $\begin{array}{l}\text { Percentil } \\
\mathbf{7 5} \%\end{array}$ & $\begin{array}{l}\text { Percentil } \\
\mathbf{2 5 \%}\end{array}$ & Mediana & $\begin{array}{c}\text { Percentil } \\
\mathbf{7 5} \%\end{array}$ \\
\hline Trabajo & 0 & 589 & 4.284 & 0 & 198 & 2.772 & 0 & 220 & 1.695 \\
\hline Transporte & 0 & 198 & 594 & 0 & 198 & 495 & 0 & 198 & 445 \\
\hline Hogar & 0 & 28 & 820 & 0 & 0 & 750 & 0 & 120 & 735 \\
\hline Recreación & 0 & 0 & 247 & 0 & 0 & 396 & 0 & 0 & 396 \\
\hline Total & 613 & 1.965 & 5.583 & 346 & 1.647 & 5.007 & 448 & 1.485 & 3.469 \\
\hline
\end{tabular}


Tabla 4. Prevalencia de nivel de actividad física según sexo y grupo de edad

\begin{tabular}{|c|c|c|c|c|c|c|c|c|}
\hline \multirow{2}{*}{$\begin{array}{l}\text { Nivel de } \\
\text { Actividad } \\
\text { Física }\end{array}$} & \multirow{2}{*}{$\begin{array}{c}\text { Prevalencia } \\
\text { Total } \\
\text { (\%) }\end{array}$} & \multicolumn{3}{|c|}{ Sexo } & \multicolumn{4}{|c|}{ Edad } \\
\hline & & $\begin{array}{c}\text { Mujeres } \\
(n=1091)\end{array}$ & $\begin{array}{l}\text { Hombres } \\
(n=444)\end{array}$ & $\mathbf{p}$ & $\begin{array}{l}<=50 a \\
(n=609)\end{array}$ & $\begin{array}{l}51-60 a \\
(n=31)\end{array}$ & $\begin{array}{c}>60 \text { años } \\
(n=357)\end{array}$ & $\mathbf{p}$ \\
\hline Bajo & 18,4 & 15,8 & 24,8 & 0,003 & 15,4 & 22,3 & 20,2 & 0,01 \\
\hline Moderado & 66 & 73,6 & 47,3 & 0,003 & 63,4 & 62,4 & 73,9 & 0,01 \\
\hline Alto & 15,6 & 10,6 & 27,9 & 0,003 & 21,4 & 15,3 & 5,9 & 0,01 \\
\hline
\end{tabular}

Tabla 5. Prevalencia de sedentarismo según nivel educacional y nivel socioeconómico

\begin{tabular}{|c|c|c|c|}
\hline \multirow[t]{2}{*}{ Nivel de Actividad Física } & \multicolumn{2}{|c|}{ Nivel Educacional } & \multirow[b]{2}{*}{ Valor $p$} \\
\hline & $\begin{array}{l}\text { Ninguno/Básico } \\
\qquad(n=322)\end{array}$ & $\begin{array}{l}\text { Secundario/ Esc. Técnica } \\
\qquad(n=1.219)\end{array}$ & \\
\hline Bajo & 16,5 & 28,3 & 0,003 \\
\hline Moderado & 67,4 & 56,8 & 0,03 \\
\hline Alto & 16,1 & 14,9 & NS \\
\hline \multirow[t]{2}{*}{ Nivel de Actividad Física } & \multicolumn{2}{|c|}{ Nivel Socioeconómico } & \\
\hline & $\begin{array}{c}\text { Bajo } \\
(n=845)\end{array}$ & $\begin{array}{l}\text { Medio } \\
(\mathrm{n}=707)\end{array}$ & Valor $\mathbf{p}$ \\
\hline Bajo & 14,7 & 22,4 & 0,003 \\
\hline Moderado & 63,9 & 66,6 & NS \\
\hline Alto & 21,4 & 11,0 & 0,003 \\
\hline
\end{tabular}

cias más altas del Nivel de Actividad Física bajo en niveles educacionales más altos. Según el NSE, puede observarse una frecuencia mayor de Nivel de Actividad Física bajo en el NSE medio a la vez que el Nivel de Actividad Física alto es significativamente más frecuente en el NSE bajo (Tabla 5).

Además pueden obtenerse del IPAQ algunos datos que ayudan a describir el hábito de actividad física, como que 38,5\% realiza actividad física en el tiempo libre ( $\sin$ diferencia por sexo o edad), $81,1 \%$ utiliza vehículo motorizado como medio de transporte en contraste con 6,2\% que usa la bicicleta (13\% de hombres y 3,5\% de mujeres); y que $66,3 \%$ camina por lo menos 10 minutos seguidos para trasladarse de un lugar a otro en un día cotidiano. Finalmente, puede destacarse el promedio de 209 minutos (desviación estándar $=$ DS = 144) diarios que se ocupan en estar sentado en un día de semana similar a los 204 minutos en promedio $(\mathrm{DS}=123)$ que se gastan en estar sentado en un día de fin de semana (es decir aproximadamente 3 horas y media al día).

\section{Discusión}

Los resultados de nuestro estudio muestran niveles elevados de niveles de actividad física bajos o moderados en la población urbana de Temuco, especialmente en las mujeres, en personas de edad avanzada y en los de NSE medio.

Es importante destacar que el instrumento de medición logra diferenciar el nivel de actividad en diferentes actividades de la vida diaria, es así como se puede ver que existe mayor gasto energético en actividades relacionadas al trabajo especialmente en los hombres, y por el contrario el gasto energético relacionado a actividades del tiempo libre es muy bajo para ambos sexos y todos los grupos de edad, lo que explicaría los altos grados de sedentarismo reportados por otros estudios que utilizan 
como medición la pregunta sobre la realización de ejercicio por al menos 30 minutos al menos tres veces a la semana y fuera del trabajo. Otra característica es que en el IPAQ se pregunta por la actividad física realizada en períodos de al menos 10 minutos, lo que permite agrupar períodos y por lo tanto, determinar con mayor exactitud los niveles de gasto energético; el parcializar la actividad física en períodos de 10 minutos es una de las medidas recomendadas para estimular la actividad física y lograr niveles óptimos que protejan a la salud ${ }^{19}$.

Al comparar nuestros resultados con los de otros estudios, se puede mencionar el realizado en 850 adultos jóvenes (entre 22 y 28 años) de Limache $^{17}$, donde se definió actividad física insuficiente cuando existiera un gasto inferior a 600 MET-min/semana según la versión corta del IPAQ reportándose una prevalencia de actividad física insuficiente de $38,3 \%$ (50,4\% en mujeres y $22,9 \%$ en hombres), más alta que el $18,37 \%$ observado en la categoría homóloga de nuestro estudio; esta diferencia puede explicarse considerando la constitución de nuestra muestra, con un promedio de edad más elevado y mayoritariamente mujeres. A nivel latinoamericano, son pocos los estudios que utilizan el IPAQ para medir el nivel de AF de la población, y de estos estudios la mayoría están dirigidos a poblaciones de niños, adolescentes, jóvenes universitarios o adultos mayores, siendo muy escasos los conducidos en población adulta. Un estudio conducido en Brasil, demostró que el NSE alto tenía niveles de inactividad física más elevados que en el NSE bajo $^{20}$, al respecto, en nuestro estudio llama la atención el mayor nivel de actividad física bajo en NSE medio, esto puede deberse a que la medición incluye las actividades relacionadas al trabajo y el transporte, que no fueron consideradas en el estudio brasileño.

Si comparamos nuestros resultados con los de otros estudios que utilizan diferentes mediciones de la AF, podemos encontrar que en un estudio realizado en la ciudad de $\mathrm{Talca}^{7}$, la prevalencia reportada de sedentarismo es de 79,9\% (77,3 en hombres y 81,3 en mujeres), siendo las diferencias entre mujeres y hombres menores a lo observado en nuestro estudio; en el citado estudio se definió como sedentaria a las personas que durante el último mes no habían practicado deportes o realizado actividad física fuera del horario de trabajo, durante 30 minutos o más cada vez, al menos 3 veces a la semana, como la mayoría de los estudios realizados en nuestro país. Un estudio realizado en la Región Metropolitana, reportó 75,6\% de sedentarismo en los hombres y $86,9 \%$ en las mujeres; otro estudio realizado en estudiantes de Medicina mostró que la inactividad física era el factor de riesgo para enfermedades crónicas más prevalente, reportándose en $88 \%{ }^{10}$; otro estudio realizado en personas sanas de 25 comunas de Santiago reportó $68,6 \%$ de sedentarismo ${ }^{9}$ y un reporte más reciente mostró $75 \%$ de inactividad física ${ }^{21}$.

A nivel latinoamericano se puede mencionar datos provenientes de estudios en la población brasileña, donde los niveles de sedentarismo son igualmente elevados, uno de ellos sobre actividad física en el tiempo libre comunica que sólo 13\% realiza actividad física por un mínimo de $30 \mathrm{mi}-$ nutos uno o más días a la semana, y sólo 3,3\% el mínimo recomendado de 30 minutos en 5 o más días a la semana; en esta población se encontró una fuerte asociación entre en nivel de actividad física y el NSE ${ }^{22}$. Otro estudio en el que evaluaron el nivel de actividad física de acuerdo a las kilocalorías gastadas por semana, también en actividades del tiempo libre, encontró una prevalencia de inactividad física de $80,7 \%{ }^{23}$. En Paraguay existen reportes de $40 \%$ de vida sedentaria en un estudio realizado en Gran Asunción ${ }^{24}$, mientras que en uno en Uruguay se reporta $28 \%$ de sedentarismo ${ }^{25}$; en Venezuela se ha reportado $70 \%$ de sedentarismo en las áreas metropolitanas ${ }^{26}$.

En otro aspecto de nuestros hallazgos, la poca realización de actividad física en el tiempo libre, el uso de vehículos motorizados como principal medio de transporte y la cantidad de horas en sedente, pueden dar cuenta de lo descrito sobre las consecuencias del rápido proceso de urbanización en los países en vías de desarrollo como el nuestro, lo que trae aparejado cambios en las tareas relacionadas al trabajo, aumento de la mecanización tanto en lo laboral como lo doméstico, disminución de actividades para disfrutar el tiempo libre y los medios de transporte disponibles ${ }^{27}$.

Son evidentes las diferencias en resultados entre los reportes a nivel nacional e internacional, los que pueden explicarse por diferencias en las poblaciones estudiadas, escalas de medición de actividad física usadas y posibles sesgos. En nuestro estudio pueden identificarse algunas debilidades o potenciales fuentes se sesgos, como el sesgo del voluntario, presente en la mayoría de los estudios de este tipo, dado a que ingresaron los sujetos 
Actividad física en población urbana - P. Serón et al

que habiendo sido seleccionados aleatoriamente, consintieron participar en el estudio, lo que probablemente produjo el desequilibrio de la muestra en cuanto a sexo y la no inclusión de sujetos del NSE más alto, esto último podría haber arrojado más evidencia sobre la asociación del hábito de AF con esta variable; por otra parte, está el posible sesgo de deseabilidad social, dado que cada vez hay más actividades de fomento de la actividad física y más información sobre los beneficios de ella, lo que hace que la población tienda a sobreestimar su percepción de gasto energético.

Finalmente, se puede concluir que, en la población estudiada, los niveles de actividad física bajos se asocian al sexo femenino, a la edad avanzada y a los niveles socioeconómicos medios, no habiendo gasto energético importante en actividades del tiempo libre. Es necesario seguir indagando sobre otras variables que se asocien a bajos niveles de actividad física, de manera de proveer información sólida que contribuya a fundamentar mejor las políticas públicas, especial atención debiera ponerse a los espacios urbanos disponibles para una vida activa.

\section{Referencias}

1. Shephard RJ, Balady GJ. Exercise as cardiovascular therapy. Circulation 1999; 99: 963-72.

2. Yusuf S, Hawken S, Ounpuu S, Dans T, Avezum A, Lanas F, et al. Effect of potentially modifiable risk factors associated with myocardial infarction in 52 countries (the INTERHEART study): case-control study. Lancet 2004; 364: 937-52.

3. Lanas F, Avezum A, Bautista Le, Díaz R, Luna M, Islam $\mathrm{S}$, et al. Risk factors for acute myocardial infarction in Latin America: the INTERHEART Latin American study. Circulation 2007; 115: 1067-74.

4. Ministerio de Salud de Chile. Resultados I Encuesta de Salud, Chile 2003. Disponible en http://epi.minsal.cl/epi/ html/invest/ENS/InformeFinalENS.pdf. [Consultado el 10 de mayo de 2007].

5. Brown W, Bauman A, Chey T, Trost S, Mummery K. Comparison of surveys used to measure physical activity. Aust N Z J Public Health 2004; 28: 128-34.

6. Hallal PC, Matsudo SM, Matsudo VK, Araujo TL, Andrade DR, Bertoldi AD. Physical activity in adults from two Brazilian areas: similarities and differences. Cad Saude Publica 2005; 21: 573-80.

7. Palomo GI, Icaza NG, Mujica EV, Núñez FL, Leiva ME,
Vásquez RM, et al. Prevalencia de factores de riesgo cardiovascular clásicos en población adulta de Talca, Chile, 2005. Rev Med Chile 2007; 135: 904-12.

8. Berrios X, Jadue L, Zenteno J, Ross MI, Rodríguez H. Prevalencia de factores de riesgo para enfermedades crónicas. Un estudio en la población general de la Región Metropolitana. 1986-1987. Rev Med Chile 1990; 118: 597-604.

9. Kunstmann S, Lira MT, Molina JC, Meruane J, Guarda E, Marchant E, et al. Riesgo de presentar un evento cardiovascular a 10 años en personas sanas: proyecto RICAR (estudio de prevención de riesgo cardiovascular de la Sociedad Chilena de Cardiología y Cirugía Cardiovascular) Proyecto RICAR. Rev Chil Cardiología 2004; 28: 13-20.

10. Mc Coll P, Amador M, Aros J, Lastra A, Pizarro A. Prevalencia de factores de riesgo de enfermedades crónicas no transmisibles en estudiantes de Medicina de la Universidad de Valparaíso. Rev Chil Pediatr 2002; 73: 478-82.

11. Organización Mundial de la Salud. Informe sobre la salud en el mundo 2002: Reducir los riegos y promover una vida sana. Disponible en http://www.who.int/ whr/2002/es/ [Consultado el 20 de mayo de 2008].

12. Brown WJ, Trost SG, Bauman A, Mummery K, Owen N. Test-retest reliability of four physical activity measures used in population surveys. J Sci Med Sport 2004; 7: 205-15.

13. Craig CL, Marshall AL, Sjostrom M, Bauman AE, Booth $\mathrm{ML}$, Ainsworth BE, et al. International physical activity questionnaire: 12-country reliability and validity. Med Sci Sports Exerc 2003; 35: 1381-95.

14. Jacoby E, Bull F, Neiman A. Cambios acelerados del estilo de vida obligan a fometar la actividad física como prioridad en la región de las Américas. Rev Panam Salud Pública 2003; 14: 223-5.

15. International Physical Activity Questionnaire. Disponible en http://www.ipaq.ki.se/downloads.htm [Consultado el 10 de enero de 2006].

16. Guidelines for the data processing and analysis of the "International Physical Activity Questionnaire". 2009. Disponible en http://www.ipaq.ki.se/scoring.htm [Consultado el 10 de enero de 2006].

17. El Nivel Socio Económico Esomar. Manual de Aplicación. 2000. Disponible en http://www.microweb.cl/idm/ documentos/ESOMAR.pdf. [Consultado el 21 de marzo de 2006].

18. Bustos P, Amigo H, Arteaga A, Acosta AM, Rona RJ. Factores de riesgo de enfermedad cardiovascular en adultos jóvenes. Rev Med Chile 2003; 131: 973-80.

19. Haskell WL, Lee IM, Pate RR, Powell KE, Blair SN, Franklin BA, et al. Physical activity and public health: updated recommendation for adults from the American 
Actividad física en población urbana - P. Serón et al

College of Sports Medicine and the American Heart Association. Circulation 2007; 116: 1081-93.

20. Reis HFCD, Ladeia AMT, Passos EC, Santos FGDO, Wasconcellos LTD, Correia LCL, et al. Prevalencia y variables asociadas a la inactividad física en individuos de alto y bajo nivel socioeconómico. Arquivos Brasileiros de Cardiologia 2009; 92: 203-8.

21. Lanas F, Potthoff S, Mercadal E, Santíbañez C, Lanas A, Standen D. Riesgo individual y poblacional en infarto agudo del miocardio: Estudio INTERHEART. Rev Med Chile 2008; 136: 555-60.

22. Monteiro CA, Conde WL, Matsudo SM, Matsudo VR, Bonsenor IM, Lotufo PA. A descriptive epidemiology of leisure-time physical activity in Brazil, 1996-1997. Rev Panam Salud Publica 2003; 14: 246-54.

23. As-Da-Costa JS, Hallal PC, Wells JC, Daltoe T, Fuchs SC, Menezes AM, et al. Epidemiology of leisure-time physical activity: a population-based study in southern Brazil. Cad Saude Publica 2005; 21: 275-82.

24. Moreno R, Páez M, Jiménez J, Figueredo R, Palacios M, Medina U, et al. Factores de riesgo de la enfermedad cardiovascular en Asunción y área metropolitana. EDUNA 1995; 76: 30

25. Curto S, Prats O, Ayesterán R. Investigación sobre factores de riesgo cardiovascular en Uruguay. Rev Med Urug 2004; 20: 61-71.

26. Pérez J, Cortés M, Henríquez F, Lira C, Chacín L. Prevalencia de Diabetes Mellitus y otros factores de riesgo cardiovascular en la región central de Venezuela: pesquiza realizada en el área metropolitana de Caracas, Valencia y Maracay. Arch Hosp Vargas 1997; 39: 123-30.

27. Popkin B. Urbanization, Lifestyle Changes and the Nutrition Transition. World Development 1999; 27: 1905-16. 